\title{
A Constructionist Philosophy of Logic
}

\author{
Patrick Allo ${ }^{1}$
}

Received: 25 October 2016/Accepted: 25 March 2017/Published online: 9 April 2017

(C) The Author(s) 2017. This article is an open access publication

\begin{abstract}
This paper develops and refines the suggestion that logical systems are conceptual artefacts that are the outcome of a design-process by exploring how a constructionist epistemology and meta-philosophy can be integrated within the philosophy of logic.
\end{abstract}

Keywords Philosophy of information - Logic and information · Constructionism · Method of abstraction · Formal methods

\section{Introduction}

The upshot of this paper is to develop and refine the suggestion that logical systems are conceptual artefacts that are the outcome of a design-process. It is part of a broader project, namely the elaboration of an informational conception of logic, and specifically explores how a constructionist epistemology and meta-philosophy (see Sect. 2) can be integrated within the philosophy of logic.

Philosophical conceptions of logic can be formulated in two ways; either as alternatives to existing accounts (mainly truth-conditional and inferential accounts of meaning and logical consequence), or as independent-less constrainedprojects in the philosophy of logic. The views I argue for in the present paper should be evaluated from the second perspective, for they elaborate on methodological

This research was funded by the European Union's Horizon 2020 research and innovation programme under the Marie Skłodowska-Curie Grant Agreement No. 657017.

Patrick Allo

patrick.allo@oii.ox.ac.uk;

http://www.logicandinformation.be

1 Oxford Internet Institute, University of Oxford, Oxford, UK 
ideas from the philosophy of information (Floridi 2011b) instead of adhering to the research-agenda and core set of concepts presupposed by more orthodox views.

The more conservative approach I hope to avoid is implicit in many "informational views" that can be found in the literature. The proposal formulated in Allo and Mares (2012) is no different: it starts from the platitude that deductively valid arguments are precisely those arguments where the content of the conclusion does not exceed the combined content of the premises, and argues that when this condition is formalised in a sufficiently flexible manner, it provides a more satisfactory interpretation of the formal semantics of certain non-classical logics, and is more friendly towards pluralist views on logic and logical consequence (Mares 2009, 2010). We should keep in mind that when a philosophical account of logic is framed as an alternative to truth-conditional and inferential views, it is tacitly formulated within the confines set by the views it intends to replace. It specifically inherits the main concepts it relies on, and the questions and challenges it should answer. A I remarked elsewhere (Allo 2015), these constraints can be used to give logical pluralists a hard time, and the reluctance to challenge these constraints can become problematic because reliance on old rules and standards may eventually prevent conceptual innovations within logic and its philosophy.

The alternative perspective I'd like to put forward is motivated by three independent insights:

First, logical systems are conceptual artefacts that are meant to be used, traditionally as a guide to correct deduction, but with a potentially broader scope of application. Like mathematical concepts and theories, logics are cognitive technologies; ${ }^{1}$ tools we rely on to improve or correct our inferential-practices. The resulting technological perspective on logic and mathematics is often put forward with a specific focus on the epistemic benefits of using (formal) languages and notations. In that case, it makes sense to think of logic as a kind of cognitive technology: a tool or set of tools used to reason more efficiently. The proposal to see logic as conceptual technology extends the scope of this picture, and emphasises that all the core notions that logical systems give a formal account of (like validity, consistency, possibility, and perhaps even meaning) should be understood as artefacts that shape deductive reasoning practices rather than as neutral descriptions or codifications of pre-existing inferential practices. As such, logic is not only a tool because it is a calculative device, but also because it is a conceptual device.

Second, a conception or philosophy of logic should primarily be evaluated by considering how well it accounts for existing practices, ${ }^{2}$ which I here understand as existing logical theorising, and much less in terms of how well it agrees with logical orthodoxy, or how successful it is at solving a given canon of logical puzzles. As

\footnotetext{
${ }^{1}$ Stenning (2002) attributes this view to Vygotsky, but current uses of the terms "cognitive technology," "cognitive artefact," or "cognitive tool" (Avigad 2015; Dutilh Novaes 2012; Gorayska and Mey 2004; De Cruz and De Smedt 2010) need not be bound to this heritage.

${ }^{2}$ Which is merely the application of the lessons from the philosophy of mathematical practice in the context of logic.
} 
such, given a changing and highly diverse scientific practice, a more flexible conception of logic is surely more desirable. ${ }^{3}$

Third, the suggestion that an informational conception of logic can be based on the core methodological components of the philosophy of information rather than on the methodological presuppositions we inherited from specific episodes in the history of philosophy (related to the foundations of mathematics, the epistemological project of logical positivism, and the rise of the philosophy of language). Specifically, this implies that the method of abstraction and a constructionist outlook on knowledge and knowledge-production can be used as a basis on which a philosophy of logic can be formulated.

This change in perspective is the basis of an informational conception of logic, and the context in which I propose to investigate a technological and designoriented understanding of logic, ${ }^{4}$ which seeks to describe logic as an informationtechnology, or as a science of the artificial concerned with the design of interfaces as put forward by Herbert Simon.

An artefact can be thought of as a meeting point, an "interface" in today's terms, between an "inner" environment, the substance and organisation of the artefact itself, and an "outer" environment, the surroundings in which it operates. If the inner environment is appropriate to the outer environment, or vice versa, the artefact will serve its intended purpose. (Simon 1996, 6).

This perspective allows us to transcend the distinction between logic as an explanatory science, as a technology, craft or art, and as pure mathematics (Toulmin 2003, 4-5). It avoids the correct but restrictive understanding of the technical role of logic as an inference engine or calculative device, and emphasises its role as conceptual technology. Logic, in this sense, is a part of the conceptual framework we use to access the world-in the sense that it tells us how to reason about the world, but also in the sense that it determines which models of the world can actually be constructed-, and is specifically that part of such conceptual frameworks that facilitates epistemic access and critical evaluation (see Sect. 4). What logic doesn't have to be on this account, is the secure foundation on which all conceptual frameworks should rest, or the codification of certain basic features of how we happen to access the world (a regimentation of a pre-theoretical conceptual framework).

When logic or logics are seen as conceptual and calculative technologies, evaluating their correctness or adequacy becomes a more complicated and more local issue. Practical concerns, like decidability and computational complexity, or

\footnotetext{
3 This view remains controversial. The majority-view is still that there is a distinction between a narrow core of Logic proper, and a wider range of formal systems that are merely logics as a matter of family resemblance. This view is strongly tied to the view that logical systems have a single, reasonably welldefined, privileged domain of application or most general purpose of logic.

${ }^{4}$ Despite the potential to develop this proposal within the confines of a Carnapian understanding of conceptual engineering, which relies on related pragmatic and pluralist considerations, I will put this option aside and avoid mixing-in (unavoidably superficial) historical scholarship in this independently motivated project. More importantly, it will keep the project within the confines of the philosophy of information rather than to turn it into a neo-Carnapian project in the philosophy of logic.
} 
even the availability of convenient proof-systems acquire more importance. This suggests that the correctness of a logic should perhaps not be reduced to how well a formal consequence relation captures what it means for a conclusion to be true in virtue of the truth of its premises. More generally, logics become associated with specific epistemic or cognitive goals and their success should then be assessed by asking how well they help us achieve these goals. Traditionally, this purpose is understood relative to a narrow goal, but with a broad scope of application, namely as correct (deductive) inference irrespective of the domain or context of application. It is, on that account, an all-purpose and hence de facto purpose-agnostic tool. This assumption isn't necessary, and it paradoxically only leads to very narrow understandings of logic. This assumption can be understood as the result of generalising the purpose-agnostic nature of how we apply natural languages to the realm of formal languages, because it ignores that "[w]e decide to use a formal language, for a specific task, but [that] no such decision seems to underlie our use of natural language." (Stokhof 2008, 600).

Putting aside many of the traditional requirements of a philosophy of logic doesn't, however, imply that the project I've outlined has to be carried out in a void. There is a rich scientific practice (what logicians do) that should be respected, or at least be taken into account. What is relevant to this practice, should in principle be relevant to a philosophical account of this practice. Being critical of logical orthodoxy doesn't have to imply that all of it's questions and presuppositions automatically become irrelevant. Rather, they should simply no longer be taken as absolute requirements.

To defend this alternative perspective on logic, I will first (Sect. 2) describe the core components of the philosophy of information, namely constructionism and the method of abstraction, and start to explain how these can be used to revise how we often think about logic and logic-choice. Next (Sect. 3), I will provide a minimal account of the role of logic that will allow me to situate the choice of a logic within the broader process of the adoption of a level of abstraction. This gets us halfway to the conclusion that logic can be seen as a knowable interface. By describing logical systems as knowable artefacts, this argument is completed. Section 4 does so by reviewing how, as a mathematical discipline, logic-design is characterised by, amongst others, the need for knowable formal languages, efficient proof-systems, and compact notation. To conclude, I summarise the constructionist philosophy of logic as the displacement of absolute or general epistemic foundations by relative epistemic foundations that can be understood in terms of reliable communication based on shared (in the strong sense of common information) conceptual resources.

\section{Constructionism and the Philosophy of Information}

Constructionism, as a general philosophical view, combines an epistemological thesis about what can be known (Floridi 2011a) with a metaphilosophical view on the nature of philosophical questions (Floridi 2013). When combined, these lead to the recommendation that philosophy should be concerned with the design or 
engineering of concepts. Below I briefly summarise the underlying argument, and then connect it to the method of abstraction.

As an epistemological thesis, constructionism counters the negative view that we cannot directly know the true nature of the world, with the positive view that we can know what we make. Given these restrictions, our best way of improving our knowledge of the world is to improve our knowledge of the means we use to access the world (Floridi 2011a, 300). These can be understood as the technological, conceptual and cognitive artefacts we use to investigate, describe and reason about the world. Floridi summarises this epistemological insight as follows:

Knowledge is not about getting the message from the world; [but] first and foremost about negotiating the right kind of communication with it. (Floridi 2011a, 284)

This account induces two essential changes in how knowledge is traditionally conceived by, first, no longer seeing the acquisition of knowledge as a passive exercise (e.g. mere observation), and, second, by affirming that indirect or mediated knowledge is — given an adequate channel or medium — genuine knowledge. Highly simplified, and without providing a full argument, constructionism then amounts to the view that given our limitations and abilities a maker's knowledge is the right kind of knowledge for the kind of epistemic agents we are (Floridi 2011a, 292). The knowledge we should pursue is knowledge that relies on the most appropriate conceptual resources. This view naturally applies to philosophical knowledge, but shouldn't be restricted to it.

As a meta-philosophy, constructionism does not constrain the scope of the knowable, but the scope of what counts as a good philosophical question. Specifically, this includes the view that philosophical questions are questions that require noetic resources (Floridi 2013, 211) - the conceptual artefacts already mentioned above-, as well as the suggestion that these resources need not be "given," but might instead have to be developed. Philosophy therefore requires conceptual analysis, but often has to be complemented with conceptual engineering or design. Supplementing conceptual analysis with conceptual engineering changes the status of the conceptual apparatus that is "given" or "inherited" in the sense that existing conceptual frameworks are treated as the outcome of a design-process, and are not granted any special authority. The intended contrast can be made more explicit by making clear how data should be understood in the context of a philosophical inquiry. If existing practices and the concepts used and presupposed by these practices are treated as the data from which conceptual analysis should start, there is little difference between respecting the data and adhering to preexisting conceptual frameworks. One can still disagree on what the data mean (Löwe and Müller 2011), but one cannot entirely ignore the data. The status that is traditionally accorded to natural language in logical analysis is an example of this view, but should be contrasted with (for instance) Stokhof's analysis of how meaning (the object of semantics) is not a natural kind, but a theoretical construct obtained from empirical elements, philosophical assumptions, and insights from other disciplines (Stokhof 2008, 598). 
If one focuses on conceptual engineering, new and old conceptual frameworks are (potentially) different ways of "making sense of the data". They can and should be compared, but need not be in agreement. On this account, the data are only related to the subject-matter or system one is reasoning about, but do not necessarily include (regimented) natural language or other pre-theoretical descriptions of that system. ${ }^{5}$ This means that pre-existing conceptual frameworks can be revised, improved or even be totally rejected without ignoring the data or assuming that the data are unreliable (developing new concepts should not be confused with cleaning up the data, which suggests that the "clean data" came first). These views undeniably challenge the traditional role of pre-theoretical conceptions of what it means to follow from, and especially the reliance on the analysis of the semantics of natural language as a means to develop a semantics of (and associated consequence relation over) formal languages. ${ }^{6}$ Instead, they come with the methodological recommendation that being explicit about what counts as data, and being clear about one's purpose (and associated requirements) are the essential preconditions for sound conceptual engineering. ${ }^{7}$ This view is consistent with the view that the conceptual resources we associate with logic should be minimal because they should also be adequate for all purposes, but does not exclude that logical systems could also be evaluated relative to more specific purposes and requirements.

Before we can investigate in further detail the implications of a constructionist epistemology and meta-philosophy for our thinking about logic, we first need a more explicit account of the relation between conceptual resources and levels of abstraction.

The method of abstraction (Fig. 1), as I use it here, is based on a rigorous distinction between the system under consideration, and a model of this system (Floridi 2008). Adopting this perspective means that in all contexts where sufficient care is needed, any talk about the properties that are attributed to the system should be preceded by the specification of the level of abstraction at which the system is analysed, and which is used to generate a model. Usually, a level of abstraction is described as a collection of observables (typed variables with their intended interpretation) that can be used to formulate a theory about the system under consideration. As such, and this will be crucial once we move on to the subject of logic, depending on the level of abstraction we adopt, more or less can be said about a system (expressivity varies with the level of abstraction), and the system (or the state of the system) can be individuated more or less finely (granularity varies with the level of abstraction).

\footnotetext{
5 Unless the system under consideration is precisely a given linguistic practice, and the goal is to obtain a formal model of that practice.

6 For a critical review of the presumed relation between logic and natural language semantics, see Glanzberg (2015).

7 Though this isn't the place to develop this view, I do believe that some forms of logical revision are the result of adhering to, on the one hand, a strict view about the goal of logic (logic is concerned with the informal practice of inference), and, on the other hand, a very liberal view about what constitutes data (or taking too much "linguistic data" at face value). The result is an unrestricted supply of potential counterexamples that can be used to challenge almost any logical principle.
} 


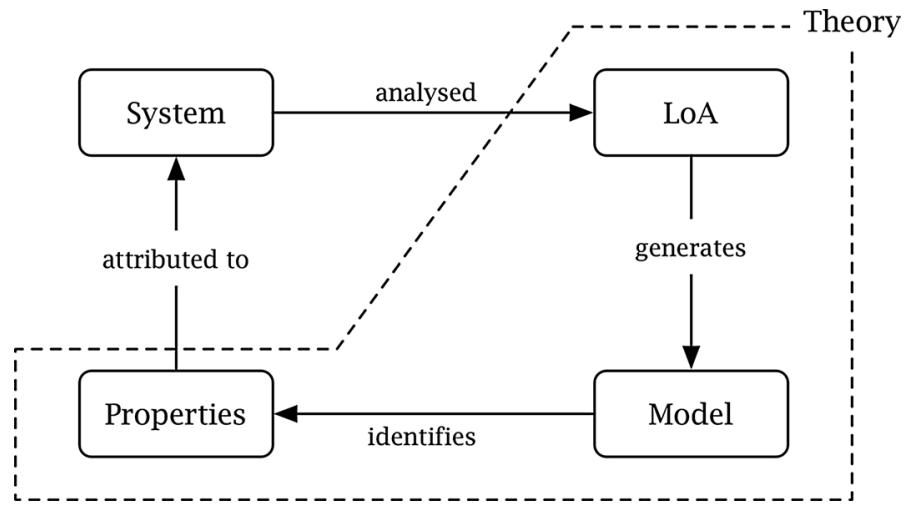

Fig. 1 The method of abstraction

The conceptual resources we have at our disposal, and the level of abstraction we adopt to analyse a system are tightly connected: without mathematical and linguistic means (typical examples of conceptual resources) we cannot give an explicit characterisation of the level of abstraction we adopt, but at the same time we can only verify whether our conceptual resources are adequate for a given purpose if we relate it to the relevant level of abstraction. Put more succinctly:

1. the distinctions we want to make (in a given context and for a given purpose) determine the conceptual resources we need (in the context and for that purpose), and

2. the conceptual resources we have determine the distinctions we can successfully make.

Understood in this way, conceptual resources are not exclusively identified with the properties we attribute to a system, or with the observables that are used to analyse the system.

Given this characterisation of how conceptual design is connected with the method of abstraction, the following double connection with logic arises. First, the question of how well a given set of (sufficiently formalised) conceptual resources allows us to capture an intended set of distinctions can be formulated as a purely logical question. As such, constructionism is associated with questions of definability and characterisation. ${ }^{8}$ Second, logic can also be seen as the most basic component of any level of abstraction in the sense that it supplies minimal conditions for what counts as a model, and which properties should be attributed to a system if other properties were already attributed to that system. Traditionally, this is how logical consequence-relations are associated with theories. Here, we could think of a logic as a minimal requirement on constructibility (an account of consistency or non-triviality), as well as a minimal set of purely formal distinctions

\footnotetext{
8 This point is emphasised in van Benthem $(2008$, 2011) where derivability and definability are repeatedly juxtaposed as the two main pillars of logic (with task-complexity as a complement).
} 
(distinctions afforded by the logical fragment of a language). This perspective is further developed in the next section.

Three morals can be derived from the above perspective. A general moral is that there is no knowledge - and no good philosophy - that is independent of a level of abstraction. This is a basic assumption that is built into the philosophy of information. It doesn't specifically influence our thinking about logic, but allows us to identify the role of logic within the philosophy of information because it implies that there are no levels of abstraction without an associated logic. The view I will challenge is that this associated logic is by definition conceptually prior to any level of abstraction we might want to adopt.

The focus on the knowable embodied by the epistemological reading of constructionism results in a different kind of connection with the philosophy of logic based on the idea that logical systems are the pinnacle of knowable conceptual frameworks. Modern formal and symbolic logics are, in several respects, designed with the intent to optimise epistemic access (to themselves, as well as to the subjectmatter they are used to reason about). This theme is developed in more detail in Sect. 4.

Stressing the need for conceptual design, and the purpose-dependence on what qualifies as adequate conceptual resources, on the other hand, leads us to the inherent pluralism of informational conceptions of logic. The relative freedom in conceptual design, together with the assumption that there is no "most general" level of abstraction or "purpose independent" conceptual framework give rise to a pluralism about levels of abstraction and conceptual resources that we cannot easily stop from spilling over into the realm of logic. This claim is developed in Sect. 3.

\section{Logics as Levels of Abstraction}

The adoption of a level of abstraction (LoA) can be understood as the selection of a collection of observables or basic relevant features of a system. From a formal perspective, this is very close to picking out a set of basic expressions; a set of atomic propositions to build a propositional language (the $p, q$, and $r$ 's), or set of predicates and constants (the $P, Q$, and $R$ 's; the $a, b$, and $c$ 's) to build a first or higher-order language. ${ }^{9}$ If this choice is made with a certain logic in the background, the selection of a set of basic expressions settles everything that can be said about a system and fixes how finely the state of a system can be characterised. Once a set of basic expressions is singled out, the set of complex expressions and their intended interpretation in terms of simpler expressions is unambiguously defined by the logic (and grammar). A simplistic example can be used to illustrate this:

A Toy Example Let $\mathrm{LoA}_{1}$ be such that the only relevant observables can be represented as the atomic propositions $p$ and $q$, whereas an alternative level of abstraction $\mathrm{LoA}_{2}$ requires the atomic propositions $p, q$, and $r$. Given classical

\footnotetext{
9 This could be further generalised to classifications in the sense of Barwise and Seligman (1997), but the more restricted setting is sufficient for present purposes.
} 
propositional logic, the languages that can be used to formulate theories about a given system or to describe different models of that system are propositional languages defined over these sets of basic propositions. The interpretation of any such sentence will then correspond to a possibly empty set of assignments of the relevant atomic propositions to truth-values. In a rudimentary and presumably familiar sense, $\mathrm{LoA}_{2}$ can then be considered more discerning than $\mathrm{LoA}_{1}$ because it can distinguish between, for instance, two possible ways in which $p$ and $q$ can jointly be true, and it can also be considered more expressive, for it can state that the two ways in which $p$ and $q$ can jointly be true differ with respect to the truth of $r$.

In the following pages the fact that a LoA, formalised as a language and an associated logic, gives rise to a space of possibilities will play a central role. In the above example this logical space is described as a set of assignments; possible states can then be pictured as lines of a truth-table, or perhaps as Carnapian statedescriptions. When moving to more expressive languages, more elaborate modeltheoretic means become more appropriate. Independently of the formalism we use, this approach thus equates the attribution of properties to a system with the situation of that system within a space of possibilities. Although a familiar modal picture thus arises, one should keep in mind that we do not thereby posit a pre-existing space of possibilities. All we need is the logical space that arises from the adoption of a level of abstraction.

Though the logic one assumes (i.e. the background logic) co-determines what can be said and what can be discerned at a given LoA (or with a certain collection of basic expressions), the choice of a logic is traditionally understood as something that precedes the adoption of a LoA. This approach is clear from our Toy Example, where the LoA's are described as language-signatures that are chosen against the background of a logic that is already presupposed and kept fixed between different levels of abstraction. This view is natural if we take into account that the method of abstraction is inspired by the field of formal methods, and hence inherits the classical mathematical foundations of that field. Within this formal framework a LoA will be deemed coherent iff it generates a model and thus gives rise to a consistent theory of the system.

Taking classical logic for granted causes certain limitations on what can and cannot be distinguished. A proponent of intuitionistic logic will for instance remark that no matter which set of observables/basic expressions we choose, we will never be able to distinguish between $\phi$ and $\neg \neg \phi$ (not-not- $\phi$ ) (for any $\phi$ in the language). A proponent of a paraconsistent logic, by contrast, will remark that (barring ad hoc modifications or reformulations) no LoA that presupposes a classical or even intuitionistic logic will ever allow us to distinguish two inconsistent theories of a system (or two states that are presumed to be distinct, but that are also obtained by attributing inconsistent properties to each state of the system). Most non-classical logics are motivated by the need to block certain inferences that are deemed problematic, but they can equally well be motivated by the need to make finer distinctions. ${ }^{10}$ Indeed, if one interprets the language-signature of $\mathrm{LoA}_{1}$ from our

\footnotetext{
10 A widely valid inverse relation between deductive strength and logical discrimination (Humberstone 2005) lies behind this idea that logical revision can be seen as trading deductions for distinctions.
} 
Toy Example relative to a paraconsistent logic, it becomes possible to distinguish two different states in which $p$ and $\neg p$ are jointly true (a $q$-case and a $\neg q$-case), or to distinguish the set of states where $p$ and $\neg p$ are jointly true from the set of states where $q$ and $\neg q$ are jointly true. The resulting additional distinctions are associated with a more fine-grained space of possibilities. If, for instance, the paraconsistent logic we use is based on a 3-valued truth-conditional semantics, it is easy to see that a truth-table presentation of this space of possibilities based on two basic propositions will now have $3^{2}$ instead of $2^{2}$ lines.

As such basic examples illustrate, the choice of a logic can be governed by considerations that are surprisingly similar to the considerations that are used to choose a set of basic observables. Both types of choices require what Stenning (2002) calls meta-level or extra-systemic reasoning; reasoning intended to find the most appropriate formulation of a problem or question. Since in either case the choices we make are related to our ability to make certain distinctions and to fudge or hide other distinctions, we can legitimately ask why the choice of a logic shouldn't itself become an integral part of the adoption of a LoA. Several semantic paradoxes moreover demonstrate that logic-choice and language-choice can become deeply entangled; for instance when certain language-constructions (like truthpredicates that unrestrictedly satisfy disquotational principles) can only be coherently adopted if a suited non-classical logic is used as well. Similar, but less controversial interdependencies between the adoption of logical and extra-logical machinery include the use of ambiguous or vague concepts, the use of concepts from certain historical scientific theories, or the use of predicates outside their original domain of application (Mares 2004). Such interdependencies provide us with good reasons to further integrate logic-choice and LoA-adoption.

The suggestion that the choice of a logic and the choice of a set of basic observables is often guided by similar considerations does not imply that they also serve identical purposes. Indeed, whereas the role of primitive expressions is primarily expressive (describing a system by attributing properties), it is worthwhile to distinguish between three distinct roles of logic, namely as an account of what is possible, of what can be distinguished, and of what follows from what. I will describe each of these below.

On most accounts logical possibility is the most liberal account of possibility. It is typically seen as a minimal requirement on what is metaphysically and epistemically possible because it is based on a single weak requirement of possibility, namely consistency. As such, logical possibility is naturally understood as a necessary, but not a sufficient condition for what is really possible, and is therefore easily understood as an absolute kind of possibility. This is not the only way in which we can understand logical possibility. First, because even an absolute notion of metaphysical possibility doesn't necessarily require an absolute or unique account of logical possibility (it could coincide with metaphysical possibility, but could also be more liberal and even be totally unconstrained). Second, and this is the view I shall defend, because any absolute account of possibility, understood as a LoA-independent constraint on the space of possibilities in which we situate a system when we attribute properties to it, would require us to know the true nature of that system. 
The resulting alternative account of possibility is based on the previously highlighted view that we always situate a system in a space of possibilities that arises from the prior adoption of a level of abstraction. As such, possibilities are afforded by the conceptual resources (observables and logical machinery) of a level of abstraction. A helpful way of understanding the reach of this proposal is based on an analogy with data as constraining affordances. Whereas data provide us with the material needed to start describing a system, but also constrain which descriptions are admissible (because they have to respect the data), levels of abstraction similarly provide the means to describe (conceptual and linguistic resources) while they also constrain which descriptions are admissible (because they have to, in a yet to be described sense, be constructible). Putting the Kantian undertone aside, one should be able to appreciate how our prior decision to place logic-choice and LoA-adoption on a par lets levels of abstraction simultaneously play an affording and a constraining role.

When a level of abstraction is based on classical logic, this constraining role can be identified with the requirement of (negation-)consistency: constructibility is simply the absence of contradiction. When this is generalised to other logical systems, constructibility has to be understood as absolute consistency or nontriviality. This generalisation is both deflationary (there is nothing to possibility beyond the existence of a model), and LoA-relative because what is non-trivial will ultimately depend on the logical and linguistic resources at hand. On this minimal account, successfully modelling a system requires us to come up with a model or with a non-empty space of possibilities in which we can situate the actual state of a model, but it doesn't necessarily require us to come up with a model that doesn't ascribe any contradictory properties to the current state of the system. In terms of theories, successful modelling then means developing a non-trivial (but possibly inconsistent) theory about the functioning of the system. In both cases the presumed standard for success is inevitably minimal, and further constraints either result from the data or from the LoA one has adopted.

The question of what is possible or constructible cannot entirely be separated from the question of when and how two possibilities or possible states of a system can be distinguished. This is because distinctions bear on the availability of sufficient possibilities, as well as on the expressive means to describe these possibilities as distinct or non-equivalent possibilities. We can look at this issue from two complementary perspectives. We can ask when two theories about a system are distinct, or we can ask when two models (or sets of models) of a system are distinct. Both perspectives are connected in the usual way: a theory can be associated with a set of models of a system, and a model or set of models can be described by a theory.

From a logical perspective, two theories are distinct if they are not logically equivalent; that is, if at least one of them cannot be deduced from the other. This criterion is incorporated in how logicians normally represent theories, for when theories are seen as deductively closed sets of statements their equivalence is reduced to their identity. If a deductively weaker logic is used, finer distinctions between theories become available. Equivalently, if two theories $\mathbf{K}$ and $\mathbf{L}$ are not equivalent, then $\mathbf{K}$ or $\mathbf{L}$ should have a model that isn't also a model of $\mathbf{L}$ or $\mathbf{K}$. This 
means that one theory should exclude a state $S$ of the system that the other theory deems possible. Consequently, the non-equivalence of $\mathbf{K}$ and $\mathbf{L}$ depends on whether such a state $S$ is constructible. ${ }^{11}$ The absence of any distinction between two inconsistent theories that rely on classical logic as their background logic illustrates this in the most radical way: since neither theory has models, neither can also have a model that isn't also a model of the other. ${ }^{12}$

Secondly, the question of whether two models are distinct refers to two potentially non-equivalent questions. It can refer to the question of whether there are two different models (can we distinguish two states of a system by constructing two different models?), or it can refer to the questions of whether two such different models also have two distinct descriptions or theories. Answers to these two questions can diverge because the language that is used to specify which models there are (the meta-language) is not necessarily the language that is used to formulate theories (the object-language). The study of how these two ways of characterising spaces of possible models converge and diverge is an essential part of logic as a mathematical discipline, and includes several central results in modeltheory. ${ }^{13}$ Here, we only need to refer to the fact that the fine distinctions that can be captured with the mathematical means assumed by a meta-language will not always be within reach of the object-language. ${ }^{14}$ As such, spaces of possible models can be more fine-grained than what can be captured by the language used to describe these models. Given the role it plays in one of the earliest theories of semantic information (Carnap and Bar-Hillel 1952), it is interesting to point out that Carnap's use of state-description closes this gap by restricting the means for characterising logical spaces to the languages used to formulate theories.

Given these two perspectives, we get a reasonably clear picture of how distinctions afforded by a logic are related to which models are constructible within that logic, and when two models can receive different descriptions.

Looking at logics as consequence-relations leads us back to the traditional core business of logic. Logic is essential to any modelling practice because it accounts for how information that is available in principle to a modeller is correctly turned into readily available and/or accessible information. Since we already granted that logical possibility and logical discrimination are LoA-dependent, we must now also concede that what counts as a logically valid deduction is also LoA-dependent. From a purely formal perspective this is barely surprising. When we reason about a system we reason about the possible states of that system that are not excluded by

\footnotetext{
11 Do note that this account applies equally well to two theories that rely on the same distinctions (possibly combined with different data), as to two theories with different levels of abstraction (and possibly the same data).

12 One could also make a similar point by just pointing out that what is true of a model is itself closed under logical consequence, but this gets the explanation backward since the characterisation of what counts as a model doesn't refer to the fact that they must have a consistent description. The latter is a fact that has to be proved by proving that the logic is sound. Put differently: constructibility implies nontriviality, but isn't to be defined in terms of it.

13 The Löwenheim-Skolem theorem and Lindström's theorem in classical first-order logic, or the van Benthem characterisation-theorem and the Hennessy-Milner theorem in modal logic.

14 This elementary points even ignores the more challenging question of which classes of models can be defined by formulae.
} 
the information we have about the system. Valid deductive reasoning, then, doesn't exclude any further possible states of the system. Conversely, because an invalid inference has a conclusion that rules out possible states of a system not already ruled out by its premises, it is an inference for which we can find a counterexample; a possible state of the system not excluded by the information we have. It is in this sense that the set of valid inferences depends on the models of a system we can successfully construct. If the latter is LoA-dependent, so will the former.

The above claim that valid deductive reasoning should not exclude states of a system not yet ruled out by the premises can be used to explain in which sense logic can be seen as a reliable informational practice or as a constraint on how we should extract information from the models we construct of a given system. When compared to traditional truth-theoretical and inferential conceptions of logic, this view does not really seem able to explain why this is sufficient for an inference to be reliable (or as a referee aptly put it, to consider the resulting formal system as a logic). There is no sense in which truth, or another epistemically valuable property like assertibility or warrant, is preserved. The absence of such a stronger ontological or epistemological grounding is continuous with the equally uncommitted sense in which earlier in this section 'successful modelling' was described in terms of the avoidance of triviality. This is a lacuna in the present proposal, but unfortunately one that I cannot satisfactorily address at this point.

What I hope to do instead is explain how the current deflated account of the constraints imposed by a logic does account for a relative type of epistemic security. ${ }^{15}$ My proposal works best in a dialogical setting based on the interaction between a Verifier and a Falsifier, or between Nature and an Inquirer. If we consider this from the first standpoint, the idea is that once a LoA is agreed upon, the fact that a valid argument is an argument for which no counterexample can be found at that LoA becomes a guarantee that if one reasons validly from what is already accepted, no counterexample can be generated or presented that wasn't already conceivable. Similarly, this ensures that no new information can be advanced by either participant that wasn't already possible. As such, agreeing on a LoA (and hence on a logic) creates a guarantee for Verifier that whatever can be put forward by Falsifier should already be in the common space of possibilities. Moving to the second standpoint, the choice of a LoA can be seen as the interface through which an Inquirer asks questions to Nature, and which provides the space of possibilities that can be used to challenge the conclusions Inquirer arrives at. On either perspective, certain types of misunderstandings and challenges are relegated to the realm of meta-level reasoning. In this sense, the epistemic security granted by a logic is identical to the epistemic security granted by a level of abstraction. When on a traditional account a contradiction can be dismissed on the ground that it cannot be true, here it can only be dismissed if at the current LoA it leads to triviality; that is, if it cannot be placed in the common space of possibilities.

When logical methods are placed in the wider perspective of modelling-practices, constructibility, logical discrimination and valid consequence all stand on the same footing. This means that there is no preferred order of explanation (e.g. the absence

\footnotetext{
$\overline{15}$ See Allo $(2017, \S 4)$ for a slightly different, but also more detailed development of this idea.
} 
of a counterexample explaining why a certain inference is valid), but also that the epistemic virtues associated with successful construction, fine logical distinctions and deductive strength do not have a natural ordering either. Trade-offs between the ability to draw fine distinctions and the ability to deduce contentful consequences have to be negotiated, but no application-agnostic way of telling which should be valued more highly can be assumed. The received view in logic, however, does seem to presuppose such epistemological and explanatory orders. This can be seen in how even proponents of non-classical logics think of giving up classically valid argument-forms as a high price to pay to avoid certain particularly unwelcome outcomes (like triviality), but it is perhaps even better illustrated by how pragmatic arguments for weaker-than-classical consequence relations or finer-than-classical (logical) distinctions are countered with claims about how accepting such logics forces one to accept metaphysically dubious entities like impossible worlds (Priest 1997).

The resulting picture of logic is one that steers clear of many historical divides and controversies (most notably between realists and anti-realists), and that adheres to few absolute principles. We have (1) formal trade-offs between what can be distinguished and what can be deduced, or between what can be expressed and what is decidable. This provides a general framework in which all logical modelling should take place. (2) Absolute consistency as the weakest notion of consistency can be accepted as a basic (and arguably unavoidable) criterion for successful construction (without models we cannot make any distinction at all). But (3) there is no underlying ontology that can guide us and help us decide which distinctions are genuine, or which inferences are truly valid. In particular, classical logic should not be preferred because it maximises the amount of deductions that can be made; conversely, the empty logic should not be adopted because it is more generally applicable than any other alternative (no matter what the data are).

\section{Logic as Cognitive Technology}

As a scientific practice the development of logic can very naturally be described as a constructionist enterprise, but the features that support this view are often ignored within the philosophy of logic. One reason for this is that most basic logical theorising is based on the conceptual analysis of a pre-theoretical grasp of what it means to "follow from" (see e.g. Rumfitt 2015, §2.1); an activity that may not necessarily require the creation of novel conceptual machinery, or at least for which no such need is perceived. The historical development of mathematics, but also of logic should cast some doubt on this view, which many textbooks in logic keep alive. A side-effect of complementing the role of conceptual analysis in logic with conceptual engineering is that "reasoning in the vernacular" loses its privileged status as a standard against which all formal accounts of deductive inference should be judged.

In this section I review a number of formal properties of logical systems that often serve as criteria for the proper behaviour of logical systems, and explain how these features support the view that logic isn't simply in the business of developing 
conceptual artefacts and calculative devices, but that it actively strives for knowable conceptual artefacts (logical systems as white boxes) and efficient calculative devices (logical systems as tools to know with). This focus on knowability and even feasible knowability is widely recognised, but the extent to which this supports a constructionist epistemology (and philosophy) of logic has never been made explicit.

Consider first the use of formal languages. The traditional story here is that formal languages allow one to avoid the ambiguity that is typically associated with natural language, or that - as is clear from the development of symbolic notation in mathematics - formal languages can serve as notational aids (Stokhof 2012, 545), and can make calculations and proof-procedures more tractable (Dutilh Novaes 2012, §3.2.3; Macbeth 2014, §6.5, 7.1). These are primarily features associated with seeing logic as a cognitive tool, but which do not yet support the idea of formal languages as knowable entities. The latter feature is instead a consequence of treating formal languages as mathematical objects that can be studied with the methods of mathematics. To anyone who ever wrote down a soundness proof by induction over the language, or relied on the ennumerability of a language as part of a Lindenbaum construction (two common examples from intermediate courses in logic), it is clear that our knowledge and insight in many logical systems derive at least in part from the fact that the language in which it is formulated has many desirable mathematical properties that are linked to its recursive structure. ${ }^{16}$ This gives us, as often in mathematics, finitary means to reason about infinite structures.

It is also worthwhile to contrast how in logic and in formal semantics formal languages are used to reformulate problems as tractable problems. As emphasised by Stokhof and van Lambalgen in their critical evaluation of modern linguistics, formal languages can be seen as idealisations that are meant to be used in the same way as abstractions are used in the sciences to "turn[...] a natural phenomenon into a 'suitable' object of scientific investigation" (Stokhof and Van Lambalgen 2011, 7). ${ }^{17}$ Here, formal languages are meant to be used as a knowable model of natural language (the object of study or system). When logic is seen as one of the tools we use when we build models, the use of formal languages (and more generally of mathematical techniques) leads to knowable tools, and it is the evaluation of these tools that as a result becomes tractable. This view allows us to retain a close connection with how logic was developed to formulate a more rigorous foundation of mathematics, but in doing so it accords more importance to rigour than to the goal of obtaining a secure foundation. As such, seeing logic as tractable cognitive technology is more concerned with facilitating access to how claims are justified (see e.g. Toulmin 2003), than with the development of absolute foundations.

The general theme that the structures defined in formal logic, like languages but also proofs, are typically built to be knowable because they are recursively defined is best understood in terms of a highly idealised notion of knowability. The

\footnotetext{
16 Note that this is a much more modest claim than the view that languages are learnable because they are compositional (Patterson 2005).

17 I here ignore the question of whether their distinction between idealisation in linguistics and abstraction in the sciences is as strict as they claim. For a critical view, see: Nefdt (2016).
} 
emphasis on tractable knowability is, by contrast, primarily associated with efficient proof-procedures. This can already be illustrated by simply looking at the diversity of proof-systems that are available for many standard logics, where we notice that while all of them are equally good if we only consider their adequacy in the abstract (i.e. they are all sound and complete), their respective virtues make them better at some tasks and worse at others. ${ }^{18}$

Such practical considerations only gain in importance as we move towards actual uses and implementations (often in software) of logical systems. This could be illustrated by looking at how tableau-systems and other cut-free calculi are optimised to perform better (D'Agostino et al. 1999; Gasquet et al. 2013), but also by the search for decidable yet expressive fragments of first-order classical logic like in Horn logic (1951) or the guarded fragment (Andréka et al. 1998).

An interesting complication of this discussion arises when two desirable features of useful proof-systems are compared, namely their computational complexity and whether they can be used in automated proof-systems because they can be implemented as a mechanised proof-search procedure. As argued in D'Agostino and Mondadori (1994), the need for mechanised procedures has often been taken to imply that only cut-free proof-systems could be used in automated theorem-provers; even if this implied accepting a substantially higher computational complexity. By recognising that mechanical proof-procedures only require the weaker sub-formula property (which is necessary, but not sufficient for the redundancy of the cut-rule), this trade-off can be resolved and both desirable features can be satisfied by the same system (as illustrated by the KE tableau-system). I highlight this specific case for two reasons. First, because it nicely illustrates the dynamics between, on the one hand, the identification of trade-offs between desirable epistemic features of logical systems, and, on the other hand, the questioning of the assumptions that cause these trade-offs. ${ }^{19}$ Second, because the sub-formula property is a good example of a property that is also desirable because it is associated with epistemologically reliable justifications in the sense that it reduces complex truths to simpler truths, and leads to self-contained proofs (Poggiolesi 2012, 445).

A less well-known epistemic virtue of formal languages in logic is their representational succinctness. This criterion was developed in the context of knowledge-representation to compare how efficiently knowledge situations can be expressed, and put forward as an additional consideration that should be taken into account in addition to the usual trade-off between expressiveness and computational complexity because it allows one to factor in the cost of translating expressions from one formal language into another (Gogic et al. 1995). Such insights also play a role for logical systems that have direct applications in certain philosophical domains. French et al. (2013), for instance, prove that Public Announcement Logic (the most basic system of Dynamic Epistemic Logic) is exponentially more succinct than

\footnotetext{
18 Axiomatic systems typically lead to cumbersome proof-procedures, but are generally easy to reason about and can often be put together in a modular way. Proof-construction and searching for proofs through heuristic rules is a lot easier in natural deduction-systems, but these will often lack other desirable properties, and be generally harder to reason about.

19 And indeed, as remarked by D'Agostino and Mondadori, the need to rely on cut-elimination makes it harder to combine different simpler results into a more complex result $(1994,287)$.
} 
epistemic logic, and thereby give a strong argument in its favour given the wellknown fact that it is not more expressive, and indeed also not more complex (Lutz 2005), than epistemic logic.

More examples could be given, but the main point of illustrating how constructionist virtues play a role in the formulation of logical systems can no doubt be appreciated with just these few examples. The moral of these examples is that concerns that are associated with tractable or feasible knowability cannot properly be accounted for in a philosophy of logic that primarily focuses on the extension of a consequence relation (what does and doesn't follow from what), or that excludes epistemic virtues that may be beneficial in specific cases because logic is meant to be application-agnostic.

\section{Conclusion}

To conclude, I'd like to come back to the suggestion from Sect. 1 that logic may be understood as an interface or as an information-technology, and explain how this perspective, on the one hand, affects the philosophy of logic, and, on the other hand, may contribute to how we think about the sciences of the artificial and perhaps even artificial intelligence.

By asking which type of interface logic provides, the views put forward in Sects. 3 and 4 can be further integrated. The main moral we may draw from seeing logics as levels of abstraction is that logic provides a reliable communication interface; something that ensures the alignment of the concepts used to describe a given system and constrains the range of admissible counterexamples. Remark, moreover, how the earlier description of how a LoA adopted by Inquirer to question Nature seamlessly fits Herbert Simon's description of an interface between an inner and outer environment.

The emphasis on how logic facilitates knowability and feasible knowability suggests, by contrast, that logic also provides an interface that is in principle - and ideally also in practice-open to scrutiny. Each of these features has clear origins in the history of (modern) logic, and can naturally be connected to the desire to reduce ambiguity and equivocation, and to the need for rigorous standards of proof (for instance in the foundations of mathematics). At the same time, they simultaneously point to two preconditions for reliable informational interaction, namely:

1. common concepts and

2. common standards for justification,

and to two preconditions for reliable epistemic scrutiny, namely:

3. common concepts (again) and

4. feasibly verifiable reasoning and justification-procedures.

These features provide a counter-point to the (intended) deflationary approach to logic that I adopted in this article, and which makes it hard to explain how logic could be used to provide any kind of absolutely secure ontological or epistemic 
foundations. They moreover sit well with a constructionist epistemology and metaphilosophy because they (1) emphasise the need to negotiate or to agree on a communication-channel, (2) imply that a reliable channel or interface should be free of noise and equivocation, (3) recognise the role of meta-level reasoning, and (4) adhere to a white-box philosophy with respect to the conceptual and cognitive technologies one has to rely on to obtain knowledge from the world.

When we thus reconsider the nature of logic from a constructionist perspective, logic becomes associated with epistemic ideals that can in principle be used to evaluate various forms of automated reasoning-systems, ranging from theoremprovers that are based on strict procedures to so-called algorithmic systems that rely on machine-learning methods. Three notable examples come to mind, and I describe them briefly as illustrations of this suggestion.

The first example concerns a notorious problem within the philosophy of mathematics, namely the acceptability of computer-generated proofs or proofs that can only be checked by a computer; for instance because it includes the verification of an excessively large set of cases. The text-book example of such a mathematical result is the proof of the 4-colour theorem, which continues to preoccupy philosophers of mathematics (Calude 2001). Here, we only need to note that the debate does not primarily concern the correctness of the result, but rather its failure to adhere to the standard of surveyability to which mathematical proofs should conform.

The second example concerns the hotly debated topic of (autonomous) algorithmic data-processing, and in particular the question of how the use of epistemically opaque forms of data-processing make the decisions based on the outcomes of such processes immune to criticism or contestation. ${ }^{20}$ Again, we only need to note how unknown epistemic standards and procedures, unknown or unintelligible concepts and categories, or simply the reliance on procedures that are too complex to reason about violate the epistemic ideals outlined above.

The third and last example may be the most surprising, and exemplifies how the use of automated proof-procedures can indeed be in agreement with the epistemic ideals we highlighted. It concerns the value that is accorded to computer-verified proofs within the Univalent Foundations and Homotopy Type Theory programme. In addition to providing a new way of thinking about the foundations of mathematics, this grand project also sets new epistemic standards for foundational work. Highly simplified, these standards combine the adherence to the very strict standards of formalised type-theory (which is made possible with the help of automated proof-assistants like Coq or Agda) with the adoption of the same high standards as the "implicit formal basis of informal reasoning" (Univalent Foundations Program 2013, 7). Again, we can remark how, this time in a fully formalised context, explicit concepts and proof-procedures together with the conceptual and technological means to efficiently use and verify these concepts and procedures can be used to certify the epistemic reliability of mathematical results. ${ }^{21}$

\footnotetext{
${ }^{20}$ See Mittelstadt et al. (2016) for a recent survey of the debate, and Hildebrandt (2016) for a discussion of the preconditions of contestation.

21 On the implications of the use of proof-assistants based on formal type-theory for the older debate on the four-colour theorem, see Arkoudas and Bringsjord (2007).
} 
Open Access This article is distributed under the terms of the Creative Commons Attribution 4.0 International License (http://creativecommons.org/licenses/by/4.0/), which permits unrestricted use, distribution, and reproduction in any medium, provided you give appropriate credit to the original author(s) and the source, provide a link to the Creative Commons license, and indicate if changes were made.

\section{References}

Allo, P. (2015). Synonymy and intra-theoretical pluralism. Australasian Journal of Philosophy, 93(1), 77-91.

Allo, P. (2017). Hard and soft logical information. Journal of Logic and Computation (Advance Article). doi:10.1093/logcom/exx010.

Allo, P., \& Mares, E. (2012). Informational semantics as a third alternative? Erkenntnis, 77(2), 167-185.

Andréka, H., Németi, I., \& van Benthem, J. (1998). Modal languages and bounded fragments of predicate logic. Journal of Philosophical Logic, 27(3), 217-274.

Arkoudas, K., \& Bringsjord, S. (2007). Computers, justification, and mathematical knowledge. Minds and Machines, 17(2), 185-202.

Avigad, J. (2015). Mathematics and language. In E. Davis \& P. J. Davis (Eds.), Mathematics, substance, and surmise: Views on the meaning and ontology of mathematics (pp. 235-55). Berlin: Springer.

Barwise, J., \& Seligman, J. (1997). Information flow: The logic of distributed systems. Cambridge tracts in theoretical computer science (Vol. 44). Cambridge: Cambridge University Press.

Calude, A. S. (2001). The journey of the four colour theorem through time. The New Zealand Mathematics Magazine, 38(3), 27-35.

Carnap, R., \& Bar-Hillel, Y. (1952). An outline of a theory of semantic information. Technical report, Cambridge, MA.

D’Agostino, M., Gabbay, D. M., Hähnle, R., \& Posegga, J. (1999). Handbook of tableau methods. Dordrecht: Kluwer Academic Publishers.

D'Agostino, M., \& Mondadori, M. (1994). The taming of the cut. Classical refutations with analytic cut. Journal of Logic and Computation, 4(3), 285-319.

De Cruz, H., \& De Smedt, J. (2010). Mathematical symbols as epistemic actions. Synthese, 190(1), 3-19.

Dutilh Novaes, C. (2012). Formal languages in logic: A philosophical and cognitive analysis. Cambridge, NY: Cambridge University Press.

Floridi, L. (2008). A defence of informational structural realism. Synthese, 161(2), 219-253.

Floridi, L. (2011a). A defence of constructionism: philosophy as conceptual engineering. Metaphilosophy, 42(3), 282-304.

Floridi, L. (2011b). The philosophy of information. Oxford: Oxford University Press.

Floridi, L. (2013). What is a philosophical question? Metaphilosophy, 44(3), 195-221.

French, T., van der Hoek, W., Iliev, P., \& Kooi, B. (2013). On the succinctness of some modal logics. Artificial Intelligence, 197, 56-85.

Gasquet, O., Herzig, A., Said, B., \& Schwarzentruber, F. (2013). Kripke's worlds: An introduction to modal logics via tableaux. New York: Springer Science \& Business Media.

Glanzberg, M. (2015). Logical consequence and natural language. In C. Caret \& O. Hjortland (Eds.), Foundations of logical consequence (pp. 71-120). Oxford: Oxford University Press.

Gogic, G., Kautz, H., Papadimitriou, C., \& Selman, B. (1995). The comparative linguistics of knowledge representation. In IJCAI (1) (pp. 862-869).

Gorayska, B., \& Mey, J. (Eds.). (2004). Cognition and technology: Co-existence, convergence, and coevolution. Amsterdam: J. Benjamins Pub.

Hildebrandt, H. (2016). Law as information in the era of data-driven agency. Mod Law Rev Modern Law Review, 79(1), 1-30.

Horn, A. (1951). On sentences which are true of direct unions of algebras. The Journal of Symbolic Logic, 16(1), 14-21.

Humberstone, I. L. (2005). Logical discrimination (pp. 207-228). Basel: Birkhuser.

Löwe, B., \& Müller, T. (2011). Data and phenomena in conceptual modelling. Synthese, 182(1), 131-148.

Lutz, C. (2005). Complexity and succinctness of public announcement logic, LTCS-Report LTCS-05-09, Chair for Automata Theory, Institute for Theoretical Computer Science, Dresden University of Technology, Germany. 
Macbeth, D. (2014). Realizing reason: A narrative of truth and knowing. Oxford: Oxford University Press.

Mares, E. (2004). Semantic dialetheism. In G. Priest, J. C. Beall, \& B. Armour-Garb (Eds.), The law of non contradiction. New philosophical essays (pp. 264-275). Oxford: Oxford University Press.

Mares, E. (2009). General information in relevant logic. Synthese, 167(2), 343-362.

Mares, E. (2010). The nature of information: A relevant approach. Synthese, 175, 111-132.

Mittelstadt, B. D., Allo, P., Taddeo, M., Wachter, S., \& Floridi, L. (2016). The ethics of algorithms: Mapping the debate. Big Data \& Society, 3(2), 1-21.

Nefdt, R. M. (2016). Linguistic modelling and the scientific enterprise. Language Sciences, 54, $43-57$.

Patterson, D. (2005). Learnability and compositionality. Mind \& Language, 20(3), 326-352.

Poggiolesi, F. (2012). On the importance of being analytic. The paradigmatic case of the logic of proofs. Logique et Analyse, 55(219), 443-461.

Priest, G. (Ed.). (1997). Special Issue on Impossible Worlds. Notre Dame Journal of Formal Logic, 38(4).

Rumfitt, I. (2015). The boundary stones of thought: An essay in the philosophy of logic. Oxford: Oxford University Press.

Simon, H. A. (1996). The sciences of the artificial (3rd ed.). Cambridge, MA: MIT.

Stenning, K. (2002). Seeing reason: Image and language in learning to think. Oxford: Oxford University Press.

Stokhof, M. (2008). Hand or hammer? On formal and natural languages in semantics. Journal of Indian Philosophy, 35(5-6), 597-626.

Stokhof, M. (2012). The role of artificial languages. In G. Russell \& D. G. Fara (Eds.), The Routledge companion to philosophy of language (pp. 544-553). New York: Routledge.

Stokhof, M., \& Van Lambalgen, M. (2011). Abstractions and idealisations: The construction of modern linguistics. Theoretical Linguistics, 37(1-2), 1-26.

Toulmin, S. E. (2003). The uses of argument, updated (edition ed.). Cambridge: Cambridge University Press.

Univalent Foundations Program, T. (2013). Homotopy Type Theory: Univalent Foundations of Mathematics. Institute for Advanced Study. https://homotopytypetheory.org/book.

van Benthem, J. (2008). Logical dynamics meets logical pluralism? Australasian Journal of Logic, 6, 182-209.

van Benthem, J. (2011). Logical dynamics of information and interaction. Cambridge: Cambridge University Press. 